\title{
Pestivirus infection in reindeer (Rangifer tarandus)
}

\author{
Magdalena Larska* \\ Department of Virology, National Veterinary Research Institute, Puławy, Poland
}

Reindeer species (Rangifer tarandus, Linnaeus, 1758) includes wild and semidomesticated ruminants belonging to Capreaolinae subfamily of Cervidae family reared in Eurasia (reindeer subspecies) and North America (caribou subspecies). Herding of reindeer has a great historical, socio-economic and ecological importance, especially to indigenous ethnic minorities. Infectious disease threats may therefore impact not solely the animal population driving it to further extinction and irreversible alterations to the wild environments of northern hemisphere, but also add to cultural changes observed as negative impact of globalization. Introduction of new technologies to control of reindeer migration between dwindling pasture areas and intensification of reindeer husbandry may facilitate the intra- and interspecies transmission of pathogens. The role of the reindeer as a potential BVDV reservoir has been studied, however, the number of publications is rather limited. The observed seroprevalences of the virus varied significantly between different geographical regions with different epidemiological situation. Most frequently limited number of animals studied and the differences in the

OPEN ACCESS

Edited by:

Thomas Passler,

Auburn University, USA

Reviewed by:

Chris Chase,

South Dakota State University, USA James Frederick Evermann,

Washington State University, USA

*Correspondence:

Magdalena Larska

m.larska@piwet.pulawy.pl

Specialty section

This article was submitted to Virology,

a section of the journal

Frontiers in Microbiology

Received: 11 July 2015 Accepted: 12 October 2015 Published: 26 October 2015

Citation:

Larska M (2015) Pestivirus infection in reindeer (Rangifer tarandus).

Front. Microbiol. 6:1187. doi: 10.3389/fmicb.2015.01187 sensitivities and specificities of the diagnostic test used could have also influenced on the differences between the studies. No pestivirus has been ever detected in free-ranging reindeer, however, a putative pestivirus strain named V60-Krefeld has been isolated from reindeer kept at a German Zoo in the 1990's. The virus was characterized as border disease virus type 2 (BDV-2) closely related to German ovine strains. The cross-neutralization studies of the semi-domesticated reindeer sera from Sweden suggested infection with a strain related to BDV-1 or BDV-2. The available data indicates that reindeer might be infected by a endemic species-specific BDV-like strain. However, the interspecies transmission of BVDV from domestic animals should not be excluded, since the susceptibility of reindeer to BVDV-1 has been confirmed under experimental conditions.

Keywords: pestivirus, BVDV, BDV, reindeer, caribou

\section{REINDEER HUSBANDRY}

Reindeer are cervids, which belong to Rangifer tarandus species (Linnaeus, 1758), subfamily Capreaolinae (New Word, telemetecarpal deer, Brookes, 1928). The worldwide population is estimated at more than four million animals of several main subspecies occupying different regions including Eurasian reindeer (R. t. tarandus) and caribou inhabiting Canada, U.S. (R. t. caribou) and Alaska (R.t. granti). Reindeer subspecies differ greatly in their size with the largest being the woodland caribou and the smallest being the wild Svalbard reindeer (R. t. platyrhynchus, 
Vrolik, 1829) from Norwegian archipelago of Svalbard (with the largest island of Spitzbergen). The circumpolar distribution of reindeer determines their diet, migration potential and reproduction specificity. As the only mammals, they are able to survive winter by feeding only on lichen (Cladonia rangiferina). They breed seasonally, however, their reproduction cycle may adapt to climate changes and access to food. The greatest threat to reindeer are predators responsible for the high mortality in particular among the youngest animals (Tryland, 2012). The alterations of the climate, which may influence winter lichen pastures lead to the necessity of supplementary feeding. Additionally, fragmentation of pasture areas disrupts natural migration routes and increases the need of transport of reindeer by lorries and usage of motor vehicles such as four-wheel drive cars, snow scooters and helicopters in their herding (Tryland, 2012). Modernization, changes of reindeer management, gathering of animals for censusing or sorting for slaughter, ear tagging, and veterinary care increases animal density and stress, and finally can lead to increased risk of transmission of pathogens (Malmfors and Wiklund, 1996).

Reindeer were reported to suffer from foot-and-mouth disease (FMD), necrobacillosis, pasteurellosis, anthrax, brucellosis, paratuberculosis, infectious keratoconjunctivitis (IKC) and contagious ecthyma (Gavier-Widen et al., 2012; Tryland, 2012). Some of the pathogens are endemic in reindeer such as Cervid herpesvirus 2 (CvHV2; das Neves et al., 2010), CvHV3 and $R$. tarandus papillomaviruses 1 and 2 (RtPV1, RtPV2) associated with IKC (Smits et al., 2013). Despite the limited number of studies on pestivirus infections in reindeer, they provide a compelling evidence of wide spread of these viruses in many populations.

\section{PESTIVIRUS EPIDEMIOLOGY IN REINDEER}

Epidemiological data of pestivirus infections in the species of reindeer are limited to only a few studies, which were performed mostly at the end of last century (Table 1). Based on specific antibody presence, pestivirus infections were confirmed in the reindeer on two continents, Europe and North America. Since in all the serosurveys bovine viral diarrhea virus type 1 (BVDV1) strains were used as a reference, the results indicated de facto BVDV seroprevalences. Only Kautto et al. (2012) have tried to establish the pestivirus strain responsible for the infections in reindeer in Sweden. The percentages of BVDV seropositive reindeer varied from $0 \%$ in the wild Svalbard reindeer of Norway (Stuen et al., 1993) and in the four populations of Swedish reindeer tested in the 1980's (Rehbinder et al., 1985) to nearly 70\% in the migratory George River caribou from Canada (Elazhary et al., 1981). The seroprevalence has been associated with variables such as herd, age, and bovine herpesvirus type 1 (BoHV1) seropositivity (Rehbinder et al., 1992; Stuen et al., 1993; Lillehaug et al., 2003; Kautto et al., 2012). The reindeer or domestic ruminant densities have not been shown to explain the variation of herd seroprevalences. The differences in the herding techniques (extensive or intensive), management or the possible presence of persistently infected (PI) animals were more likely risk factors (Kautto et al., 2012). Similarly to many other infectious diseases, the risk of infection increased with the age, resulting in a significantly higher pestivirus seroprevalence in adult reindeer in comparison to calves (Stuen et al., 1993; Kautto et al., 2012). The possible interactions between pestiviruses and alphaherpesviruses has been observed in reindeer (Stuen et al., 1993; Tryland et al., 2005; Kautto et al., 2012), which by analogy to cattle (Kampa et al., 2009), could reflect the immunosuppressive nature of both virus groups.

The questions on the pestivirus strain and the source of infection in reindeer have been raised by some authors. Except for Kautto et al. (2012), only serological test based on BVDV-1 strains isolated from cattle were used. However, it is very likely that, as in case of alphaherpesviruses, the pestivirus circulating in reindeer is specific to the species (das Neves et al., 2010). The Swedish group (Kautto et al., 2012) have found that BVDV ELISA positive reindeer sera reacted with the highest titers to border disease virus 1 (BDV-1) 137/4 strain and with relatively lower titers to BDV-2 V-60 strain isolated from reindeer at German Zoo (Becher et al., 1999, 2003; Avalos-Ramirez et al., 2001). Some cross-reactivity toward BVDV-1 NADL strain was also observed, however, it was connected to the wider crossreactivity of BVDV-1 (Becher et al., 2003). The study failed to demonstrate, which type was actually circulating in the reindeer population studied, however, it suggested BDV strain rather than a bovine pestivirus. Another convincing argument for independent infection process among reindeer and caribou was the lack of direct contact with domestic ruminants discussed by Elazhary et al. (1981) and Kautto et al. (2012), respectively. Rehbinder et al. (1992) concluded that closer contact of reindeer with domestic ruminants has not affected BVDV seroprevalence. The transmission of BVDV between cattle and reindeer by blood feeding flies has been suggested (Rehbinder et al., 1992). The virus was detected in the insects fed on PI calves (Tarry et al., 1991), however, the exposed BVDV naïve calves remained virusfree and did not seroconvert throughout the study (Chamorro et al., 2011). The transmission from cattle to reindeer was also found unlikely in Norway. The highest pestivirus seroprevalence was found in the extreme northern county of Finnmark where the density of domestic ruminants is very low (Stuen et al., 1993; Lillehaug et al., 2003). Additionally, most cattle in northern Scandinavia where reindeer are reared remains BVDV free after successful eradication plans implemented already in 1990's in Norway (Løken and Nyberg, 2013), Sweden (Hult and Lindberg, 2005), and Finland (Rikula et al., 2005).

\section{PESTIVIRUS ISOLATION AND PATHOGENICITY IN REINDEER}

The susceptibility of reindeer to BVDV-1 infection has been experimentally shown (Morton et al., 1990). The clinical signs included mucous and bloody diarrhea, transient laminitis, and coronitis, leucopenia, serous to mucopurulent nasal discharge. However, no pestivirus has been isolated from wild or semi-domesticated reindeer (Rehbinder et al., 1985; 


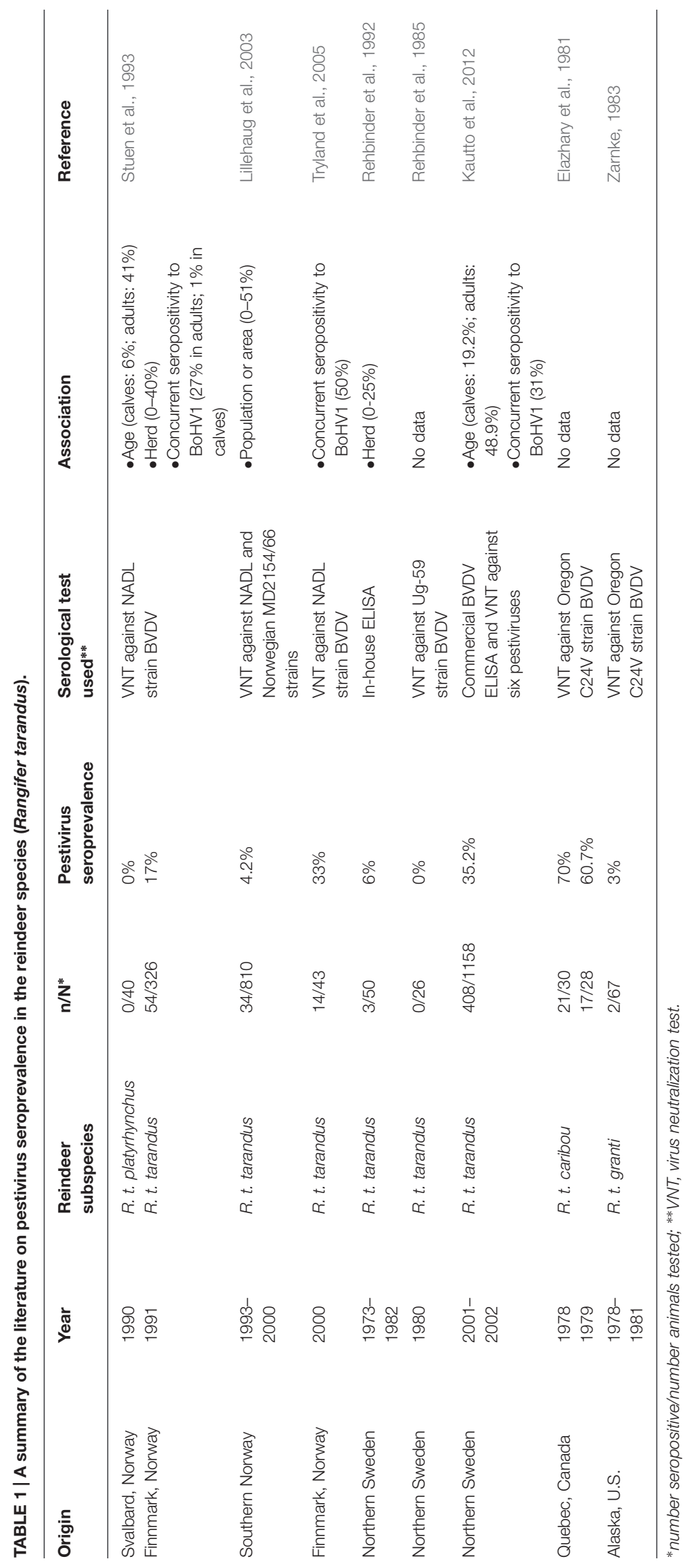


Rockborn et al., 1990; Kautto et al., 2012). The cases of conjunctivitis, ulcerative and necrotizing lesions of nose and oral mucosa in Swedish reindeer could not have been linked to BVDV infection (Rehbinder et al., 1985; Rockborn et al., 1990). The presence of pestivirus genome was also investigated in nearly 280 healthy slaughtered seronegative reindeer from the districts with up to $98 \%$ of virus seroprevalence using pan-pestivirus real-time RT-PCR (Kautto et al., 2012). No viral RNA was found in the sera of those animals.

So far the only pestivirus isolated from $R$. tarandus species was V60-Krefeld (Reindeer-1) strain obtained from reindeer, which died with signs of severe diarrhea and anorexia at Duisburg Zoo in Germany in 1996 (Becher et al., 1999; Giangaspero et al., 2006). The outbreak affected all reindeer in the zoo herd as well as an European bison (Bison bonasus) from which another, closely related strain V65-Krefeld (Bison-1) was isolated (Becher et al., 1999; Giangaspero et al., 2006). Initially, the reindeer strain has been included as a separate novel species within the genus Pestivirus (Avalos-Ramirez et al., 2001). Further phylogenetic studies based on $\mathrm{N}^{\text {Pro }}$ and E2 coding sequences revealed that V60 and V65 strains were most closely related to border disease virus type $2(\mathrm{BDV}-2)$ strains isolated from German sheep in 1999 and 2000 (Becher et al., 1999, 2003). The V60 strain grew efficiently in Madin-Darby bovine kidney (MDBK) cells with titers reaching $10^{6}-10^{7}$ TCID $_{50}$ after four consecutive passages (Giangaspero et al., 2006). The antiserum

\section{REFERENCES}

Avalos-Ramirez, R., Orlich, M., Thiel, H.-J., and Becher, P. (2001). Evidence for the presence of two novel pestivirus species. Virology 286, 456-465. doi: 10.1006/viro.2001.1001

Becher, P., Avalos-Ramirez, R., Orlich, M., Cedillo Rosales, S., Konig, M., Schweizer, M., et al. (2003). Genetic and antigenic characterization of novel pestivirus genotypes: implications for classification. Virology 311, 96-104. doi: 10.1016/S0042-6822(03)0 0192-2

Becher, P., Orlich, M., Kosmidou, A., Konig, M., Baroth, M., and Thiel, H. J. (1999). Genetic diversity of pestiviruses: identification of novel groups and implications for classification. Virology 262, 64-71. doi: 10.1006/viro.199 9.9872

Chamorro, M. F., Passler, T., Givens, M. D., Edmondson, M. A., Wolfe, D. F., and Walz, P. H. (2011). Evaluation of transmission of bovine viral diarrhea virus (BVDV) between persistently infected and naive cattle by the horn fly (Haematobia irritans). Vet. Res. Commun. 35, 123-129. doi: 10.1007/s11259010-9453-7

das Neves, C. G., Roth, S., Rimstad, E., Thiry, E., and Tryland, M. (2010). Cervid herpesvirus 2 infection in reindeer: a review. Vet. Microbiol. 143, 70-80. doi: 10.1016/j.vetmic.2010.0 2.015

Elazhary, M. A., Frechette, J. L., Silim, A., and Roy, R. S. (1981). Serological evidence of some bovine viruses in the caribou (Rangifer tarandus caribou) in Quebec. J. Wildl. Dis. 17, 609-612. doi: 10.7589/0090-3558-1 7.4 .609

Gavier-Widen, D., Duff, J. P., and Meredith, A. (2012). Infectious Diseases of Wild Mammals and Birds in Europe. Oxford: Wiley-Blackwell.

Giangaspero, M., Harasawa, R., Muschko, K., and Büttner, M. (2006). Characteristics of the 5 'untranslated region of wisent (Bison bonasus) and reindeer (Rangifer tarandus) pestivirus isolates. Vet. Ital. 42, 165-172.

Hult, L., and Lindberg, A. (2005). Experiences from BVDV control in Sweden. Prev. Vet. Med. 72, 143-148. against V60 strain preferably neutralized BDV-like and classical swine fever virus (CSFV) strains, but did not react with BVDV1 strains NY-1 and C86 (Avalos-Ramirez et al., 2001; Becher et al., 2003). If the pestivirus isolated from zoo reindeer is related to the virus circulating among free-living animals, which is very likely judging by the results of Kautto et al. (2012), the seroprevalences estimated using tests based on BVDV-1 strains may be underestimated.

In conclusion, the few reports on the distribution of pestivirus in reindeer has shown great variation of seroprevalences depending on the time of the study, geographical origin, animal age, health status, and epidemiological situation of other endemic infections. Pestivirus infection in freeranging reindeer is more likely to be independent from domestic ruminants, mainly due to the circumpolar distribution of reindeer and therefore limited contact between the species, or pestivirus eradication in far animals as in Scandinavia. Some studies suggest that the pestivirus strain circulating in reindeer is probably a species-specific BDV-like virus.

\section{FUNDING}

The author thanks National Veterinary Research Institute for funding this publication.

Kampa, J., Alenius, S., Emanuelson, U., Chanlun, A., and Aiumlamai, S. (2009). Bovine herpesvirus type 1 (BHV-1) and bovine viral diarrhoea virus (BVDV) infections in dairy herds: self-clearance and the detection of seroconversions against a new atypical pestivirus. Vet. J. 182, 223-230. doi: 10.1016/j.tvjl.2008.0 7.006

Kautto, A. H., Alenius, S., Mossing, T., Becher, P., Belák, S., and Larska, M. (2012). Pestivirus and alphaherpesvirus infections in Swedish reindeer (Rangifer tarandus tarandus L.). Vet. Microbiol. 156, 64-71. doi: 10.1016/j.vetmic.2011.1 0.018

Lillehaug, A., Vikøren, T., Larsen, I. L., Akerstedt, J., Tharaldsen, J., and Handeland, K. (2003). Antibodies to ruminant alpha-herpesviruses and pestiviruses in Norwegian cervids. J. Wildl. Dis. 39, 779-786. doi: 10.7589/00903558-39.4.779

Løken, T., and Nyberg, O. (2013). Eradication of BVDV in cattle: the Norwegian project. Vet. Rec. 172, 661. doi: 10.1136/vr.1 01525

Malmfors, G., and Wiklund, E. (1996). Pre-slaughter handling of reindeer effects on meat quality. Meat Sci. 43, 257-264. doi: 10.1016/0309-1740(96)0 0070-8

Morton, J. K., Evermann, J. F., and Dietrich, R. A. (1990). Experimental infection of reindeer with bovine viral diarrhea virus. Rangifer 10, 75-77. doi: 10.7557/2.1 0.2 .797

Rehbinder, C., Belák, S., and Nordkvist, M. (1992). A serological, retrospective study in reindeer on five different viruses. Rangifer 12, 191-195. doi: 10.7557/2.12.3.1044

Rehbinder, C., Nordkvist, M., Moreno, J. W., and Islam-ud-din Siddiqui. (1985). A suspected virus infection of the oral mucosa in Swedish reindeer (Rangifer tarandus L). Rangifer 5, 22-31. doi: 10.7557/2. 5.2 .544

Rikula, U., Nuotio, L., Aaltonen, T., and Ruoho, O. (2005). Bovine viral diarrhoea virus control in Finland 1998-2004. Prev. Vet. Med. 72, 139-142. doi: 10.1016/j.prevetmed.2005.08.010

Rockborn, G., Rehbinder, C., Klingeborn, B., Leffler, M., Klintevall, K., Nikkilä, T., et al. (1990). The demonstration of a herpesvirus, related to bovine herpesvirus 
1 , in reindeer with ulcerative and necrotizing lesions of the upper alimentary tract and nose. Rangifer 3, 373-384. doi: 10.7557/2.10.3.882

Smits, S. L., Schapendonk, C. M., van Leeuwen, M., Kuiken, T., Bodewes, R., Stalin Raj, V., et al. (2013). Identification and characterization of two novel viruses in ocular infections in reindeer. PLoS ONE 8:e69711. doi: 10.1371/journal.pone.00 69711

Stuen, S., Krogsrud, J., Hyllseth, B., and Tyler, N. J. C. (1993). Serosurvey of three virus infections in reindeer in northern Norway and Svalbard. Rangifer 13, 215-219. doi: 10.7557/2.13.4.1120

Tarry, D. W., Bernal, L., and Edwards, S. (1991). Transmission of bovine virus diarrhea virus by blood feeding flies. Vet. Rec. 128, 82-84.

Tryland, M. (2012). Are we facing new health challenges and diseases in reindeer in Fennoscandia? Rangifer 32, 35-47. doi: 10.7557/2.3 2.1.2279

Tryland, M., Mørk, T., Ryeng, K. A., and Sørensen, K. K. (2005). Evidence of parapox-, alphaherpes- and pestivirus infections in carcasses of semi-domesticated reindeer (Rangifer tarandus tarandus) from Finnmark, Norway. Rangifer 25, 75-83. doi: 10.7557/2.2 5.2.255

Zarnke, R. L. (1983). Serologic survey for selected microbial pathogens in Alaskan wildlife. J. Wildl. Dis. 19, 324-329. doi: 10.7589/0090-3558-19.4.324

Conflict of Interest Statement: The author declares that the research was conducted in the absence of any commercial or financial relationships that could be construed as a potential conflict of interest.

Copyright $\odot 2015$ Larska. This is an open-access article distributed under the terms of the Creative Commons Attribution License (CC BY). The use, distribution or reproduction in other forums is permitted, provided the original author(s) or licensor are credited and that the original publication in this journal is cited, in accordance with accepted academic practice. No use, distribution or reproduction is permitted which does not comply with these terms. 